\title{
Comment on "Correlation between Porosity and Electrical-Mechanical Properties of Carbon Nanotube Buckypaper with Various Porosities"
}

\author{
Amit Rawal, ${ }^{1}$ Ákos Kukovecz, ${ }^{2,3}$ and Vijay Kumar ${ }^{4}$ \\ ${ }^{1}$ Department of Textile Technology, Indian Institute of Technology Delhi, Hauz Khas, New Delhi, India \\ ${ }^{2}$ Department of Applied and Environmental Chemistry, University of Szeged, Rerrich Bela ter 1, 6720 Szeged, Hungary \\ ${ }^{3}$ MTA-SZTE “Lendület” Porous Nanocomposites Research Group, Rerrich Bela ter 1, 6720 Szeged, Hungary \\ ${ }^{4}$ University of Borås, 50190 Borås, Sweden
}

Correspondence should be addressed to Amit Rawal; amitrawal77@hotmail.com

Received 17 June 2016; Revised 15 September 2016; Accepted 26 September 2016

Academic Editor: Albert Nasibulin

Copyright (C) 2016 Amit Rawal et al. This is an open access article distributed under the Creative Commons Attribution License, which permits unrestricted use, distribution, and reproduction in any medium, provided the original work is properly cited.

With great interest we read a recently published article entitled "Correlation between Porosity and ElectricalMechanical Properties of Carbon Nanotube Buckypaper with Various Porosities" authored by Ling Liu and colleagues in Journal of Nanomaterials [1]. There are certain concerns and issues that question the methodology, reliability of results, and related analysis. One of the key concerns is that the authors have prepared three sets of samples of randomly aligned multiwalled carbon nanotubes (MWCNTs) in the form of buckypapers (BPs) with IDs $1^{\#}$ BPs, $2^{\#}$ BPs, and $3^{\#} \mathrm{BPs}$, having exceptionally low levels of porosity, that is, $11.3 \%, 21.1 \%$, and $39.3 \%$, respectively. The porosity $(\xi)$ and fibre volume fraction $\left(V_{f}\right)$ of any porous material are related to each other, as shown in the following equation [2]:

$$
\xi=1-V_{f}
$$

Based upon the above equation, $V_{f}$ of sample IDs $1^{\#}$ BPs, $2^{\#}$ BPs, and $3^{\#}$ BPs are $88.7 \%, 78.9 \%$, and $60.7 \%$, respectively. Such values of $V_{f}$ can be obtained neither through any experimental route for fabricating randomly aligned carbon nanotubes (CNTs) nor from theoretical perspective. To prove this point, we hypothetically analysed the maximum fibre volume fraction $\left(V_{\mathrm{fm}}\right)$ of BPs through the work of Pan et al. [3] dealing with the fibrous network that also includes the case of randomly oriented fibres. Considering each CNT as a fibre in the network $[4,5]$, the fibrous network consists of three basic segments, namely, the mean length (separation distance) between the centres of two adjacent fibres $(\bar{b})$, the mean length of contacts $\left(\bar{b}_{b}\right)$, and the mean free fibre length $\left(\bar{b}_{f}\right)$ (see [3] for details). Hence, the proportions of free length of fibre $(m)$ and that of contact length $(n)$ are given below:

$$
\begin{gathered}
m=\frac{\bar{b}_{f}}{\bar{b}}, \\
n=\frac{\bar{b}_{b}}{\bar{b}},
\end{gathered}
$$

$$
m+n=1 .
$$

Theoretically, $m \geq 0$; in case this inequality is violated; the free fibre length between the contacts will not exist, which eventually leads to $V_{\mathrm{fm}}$. Based upon these considerations, Pan et al. [3] have formulated the general relationship between $V_{\mathrm{fm}}$ and the orientation distribution of fibres, as shown below:

$$
\begin{aligned}
& V_{\mathrm{fm}}<\frac{\pi}{8 R I}, \\
& I=\int_{0}^{\pi} d \theta \int_{0}^{\pi} J(\theta, \varphi) \sin \theta \Omega(\theta, \varphi) d \varphi,
\end{aligned}
$$




$$
\begin{aligned}
& J(\theta, \varphi)=\int_{0}^{\pi} d \varphi^{\prime} \int_{0}^{\pi} \sin \chi \Omega\left(\theta^{\prime}, \varphi^{\prime}\right) \sin \theta^{\prime} d \theta^{\prime} \\
& \sin \chi \\
& \quad=\left[1-\left\{\cos \theta \cos \theta^{\prime}+\sin \theta \sin \theta^{\prime} \cos \left(\varphi-\varphi^{\prime}\right)\right\}^{2}\right]^{1 / 2}, \\
& R=\int_{0}^{\pi} d \theta \int_{0}^{\pi} d \varphi \Omega(\theta, \varphi) J^{\prime}(\theta, \varphi) \sin \theta \\
& J^{\prime}(\theta, \varphi)=\int_{\theta_{1}^{\prime}}^{\theta_{2}^{\prime}} d \theta^{\prime} \int_{\varphi_{1}^{\prime}}^{\varphi_{2}^{\prime}} d \varphi^{\prime} \Omega\left(\theta^{\prime}, \varphi^{\prime}\right) \frac{1}{\sin \chi} \sin \theta^{\prime} \\
& \pi-\sin ^{-1}\left(\frac{1}{s}\right)>\chi>\sin ^{-1}\left(\frac{1}{s}\right)
\end{aligned}
$$

where $I$ is an orientation parameter defining the orientation characteristics of fibres in the assembly, $\chi$ is the angle between the two axes of fibres having defined types of orientation distributions $\Omega(\theta, \varphi)$ and $\Omega\left(\theta^{\prime}, \varphi^{\prime}\right)$, and $s$ is the aspect ratio.

Considering the BP as a two-dimensional (2D) random network of CNTs and hence the value $I$ as $2 / \pi$ [3] whereas the expression of $R$ is given below [3],

$$
R=\frac{1}{\pi} \ln \cot ^{2} \frac{\arcsin (1 / s)}{2} .
$$

Thus, a relationship can be obtained between $V_{\mathrm{fm}}$ and $s$ of CNTs for a 2D random network, as illustrated in Figure 1. Now considering the extreme values of MWCNT diameter and length leading to minimum aspect ratio presented in [1], it can be seen that the maximum diameter and minimum length of CNT are $15 \mathrm{~nm}$ and $5 \mu \mathrm{m}$, respectively, that results in minimum aspect ratio of 333 , which inevitably leads to $V_{\mathrm{fm}}$ of $15 \%$. Therefore, porosity of BPs based upon (1) cannot be less than $85 \%$ whereas the prepared samples of [1] have a porosity ranging from 11.3 to $39.3 \%$, which does not seem rationally correct.

Since the authors have related porosity of BP to the tensile properties, the whole analysis appears to be spurious as there is no direct relationship between tensile strength and porosity (see Figure 7b of [1]). Specifically, sample $2^{\#}$ BPs having higher porosity (lower fibre volume fraction) in comparison to sample $1^{\#}$ BPs shows relatively higher tensile strength. Although the authors of [1] have claimed in "Abstract" and "Conclusion" that the tensile strength and modulus of BPs reduced with the increase in porosity. Furthermore, the authors of [1] stated "On one hand, high conductivity of BPs results from high alignment and high density (such as $1.39 \mathrm{~g} / \mathrm{cm}^{3}$ ) of CNTs [16], while the BPs in our study are porous and the CNTs are randomly stacked in the BPs with low density $\left(0.3-0.4 \mathrm{~g} / \mathrm{cm}^{3}\right)$." Here, the authors have compared their results with "ref. [16]," while this reference is related to the preparation of $\mathrm{BP}$ samples with densities ranging from 0.62 to $0.83 \mathrm{~g} / \mathrm{cm}^{3}$. It was not clear how such a claim was made related to the comparison of BP densities with those given in the literature.

In addition, we postulate possible rationale for the mentioned porosity discrepancies as a result of the limited

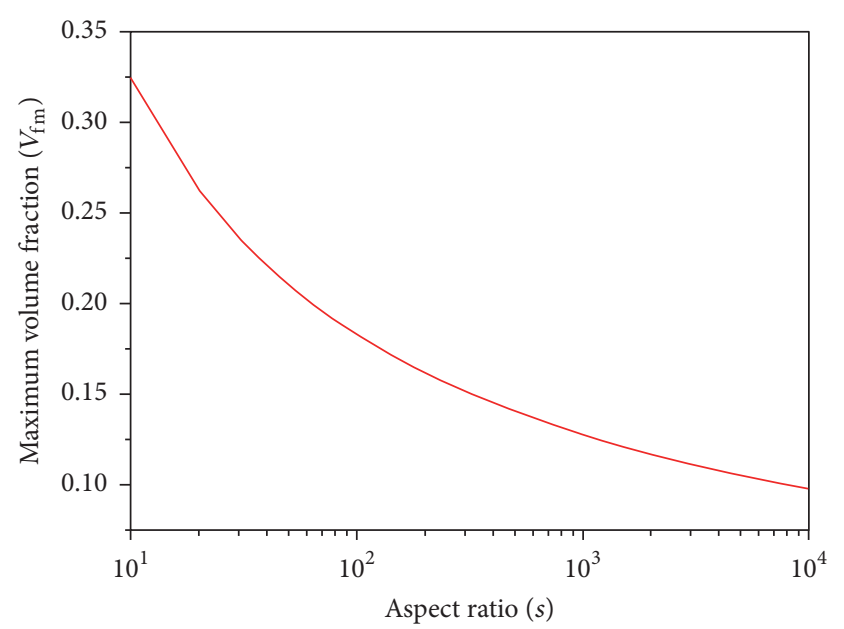

FIGURE 1: Relationship between maximum volume fraction $\left(V_{\mathrm{fm}}\right)$ and aspect ratio (s) of CNTs for a 2D random network.

applicability of the Barrett-Joyner-Halenda (BJH) method in this particular case. This method assumes cylindrical pore openings and uses the Kelvin equation to convert adsorbed $\mathrm{N}_{2}$ amount into filled pore volume. In contrast to the data reported in Table 2 of [1], it is impossible to calculate porosity directly by the $\mathrm{BJH}$ method. Rather, the calculation provides the total volume of meso- and macropores accessible to nitrogen, and this value can be converted into porosity if the total volume of the BP is known. On one hand, it is difficult to determine the latter quantity accurately because measuring the real thickness of a BP is a nontrivial task [6]. However, the inaccuracy of the pore volume as calculated by the $\mathrm{BJH}$ method represents an even larger source of potential error because of the following reasons:

(i) The BJH method underestimates the contribution of small $(<7 \mathrm{~nm}$ in diameter) pores to the total pore volume considerably (by $20-30 \%$ depending on the pore structure). According to Figure 4 of [1], such pores dominate $1^{\#}$ BPs.

(ii) Very large $(>100 \mathrm{~nm}$ in diameter) pores are considered in the pore size distribution histograms of $2^{\#}$ and $3^{\#}$ BPs. The information about such pores is found in the $p_{\text {rel }}>0.98$ region of the $\mathrm{N}_{2}$ adsorption isotherm. While this is within the manufacturerspecified measurement range of the state-of-the-art instrument used by the authors, it is well known that measuring so close to the saturation vapour pressure can be subject to considerable error even when measured with the right instrument.

(iii) Combining the previous two observations indicates that the total pore volume (and consequently the porosity) of $1^{\#}$ BPs could be underestimated and that of $2^{\#}$ and $3^{\#}$ BPs could be overestimated simultaneously.

(iv) Even though a cylindrical pore with a circular opening is a core assumption of the BJH model, the pores in the studied BPs are obviously noncylindrical as shown in Figure 2 of [1]. Therefore, all conclusions 
based on BJH calculations are necessarily limited in validity. Moreover, the pore shape appears to change from slit-like $1^{\#}$ BPs to open rectangular $3^{\#}$ BPs as the filtration pressure is modified. Therefore, the pore volume error made by using the cylindrical shape assumption is not even constant: it could vary from sample to sample.

It should be noted that most of these concerns could be resolved by showing the primary $\mathrm{N}_{2}$ adsorption-desorption isotherm data.

While the following points are not directly related to the main criticism of this letter which is about the porosity issue, we mention them because they may affect the interpretation of the results. The authors conclude from the Raman $I_{D} / I_{G}$ ratio of approximately 1.50 measured on the functionalized CNTs that the "integrity of the CNTs was not destroyed after oxidation treatment..." Actually, single $I_{D} / I_{G}$ values are of limited use in CNT Raman spectroscopy. Meaningful conclusions about the preservation of CNT integrity can only be made by comparing the $I_{D} / I_{G}$ ratio of the samples before and after the oxidation treatment, yet the Raman spectrum of the untreated sample is not presented in the paper. Further, the discussion of Figure 6a of [1] suggests that the TEM image depicts actual CNT contacts as they are found in the BP. However, BP cannot be imaged by TEM directly. Rather, it is typically sonicated in a solvent and then nanotubes are drop-casted onto a TEM grid for observation. This procedure deconstructs the BP completely. Assuming that a similar procedure was followed here, since the paper fails to specify the actual sample preparation procedures, it becomes clear that the findings in Figure $6 a$ of [1] have little actual relevance for modelling the contact network in BP. But the authors of [1] stated "The TEM micrograph (Figure 3(c)) suggested that some CNTs kept relatively good structural integrity after chemical functionalization." In such a case, it was not clear how "structural integrity" and "defects" were detected from TEM micrograph since the "structural integrity" is being pointed at a CNT with bent surface whereas the CNT with "defect" also exhibits bent surface. In addition, conduction paths depicted in Figure 6 of [1] are of course possible, but there are several other possibilities, which were not considered by the authors.

The above remarks are intended to point out the fact that the experimental data of BP and related analysis should be carried out primarily through "objectivity."

\section{Competing Interests}

The authors declare that they have no competing interests.

\section{References}

[1] L. Liu, Q. Yang, and J. Shen, "Correlation between porosity and electrical-mechanical properties of carbon nanotube buckypaper with various porosities," Journal of Nanomaterials, vol. 2015, Article ID 945091, 9 pages, 2015.

[2] A. Rushton, A. S. Ward, and R. G. Holdich, Solid-Liquid Filtration and Separation Technology, John Wiley \& Sons, Weinheim, Germany, 2000.
[3] N. Pan, J. Chen, M. Seo, and S. Backer, "Micromechanics of a planar hybrid fibrous network," Textile Research Journal, vol. 67, no. 12, pp. 907-925, 1997.

[4] S. V. Lomov, L. Gorbatikh, and I. Verpoest, "A model for the compression of a random assembly of carbon nanotubes," Carbon, vol. 49, no. 6, pp. 2079-2091, 2011.

[5] A. Rawal and V. Kumar, "Compressibility of highly porous network of carbon nanotubes," Applied Physics Letters, vol. 103, no. 15, Article ID 153103, 5 pages, 2013.

[6] V. Kumar, H. Haspel, K. Nagy, A. Rawal, and A. Kukovecz, "Leveraging compressive stresses to attenuate the electrical resistivity of buckypaper," Carbon, vol. 110, pp. 62-68, 2016. 

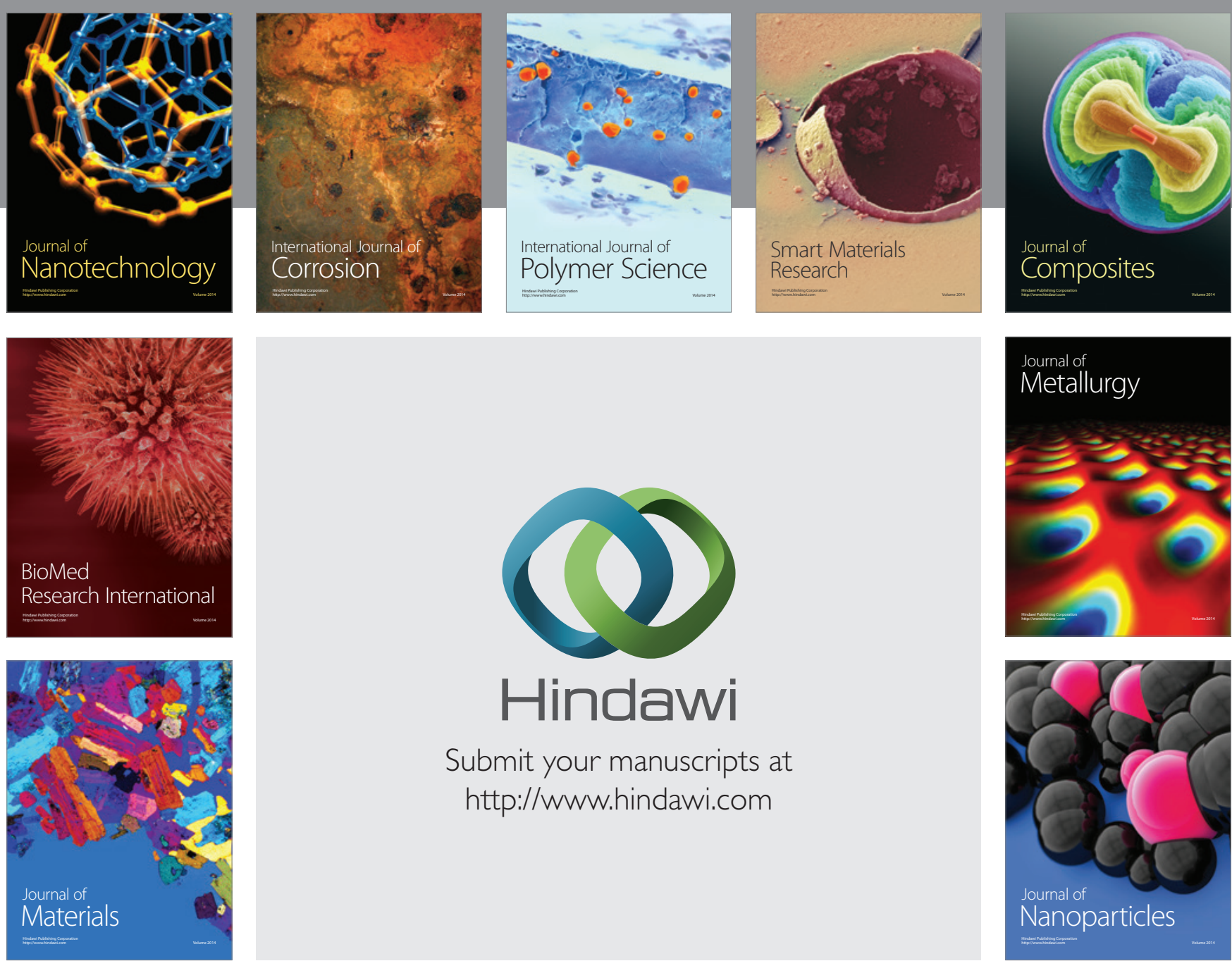

\section{Hindawi}

Submit your manuscripts at

http://www.hindawi.com

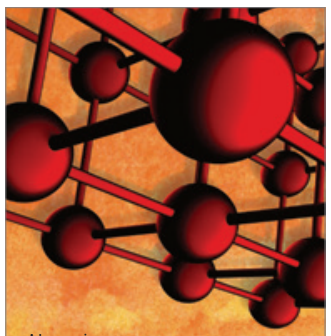

Materials Science and Engineering
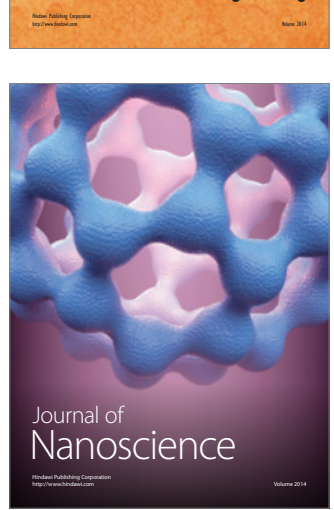
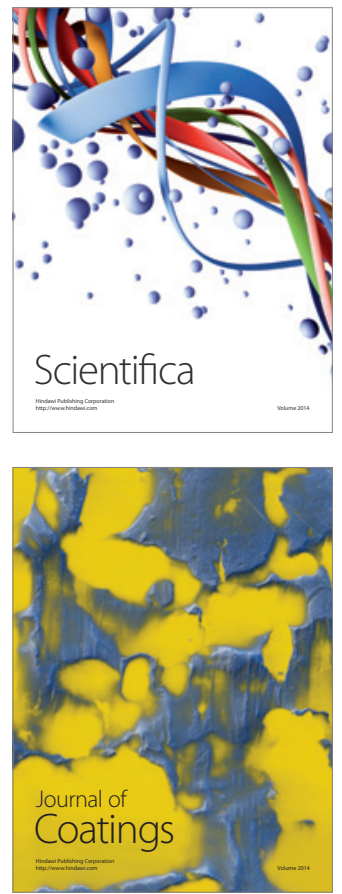
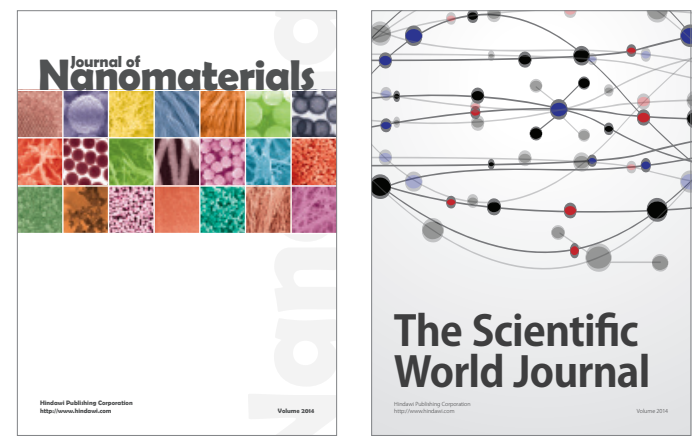

The Scientific World Journal
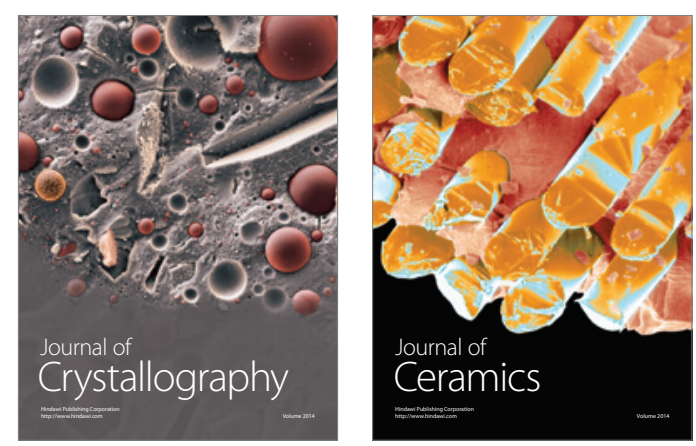
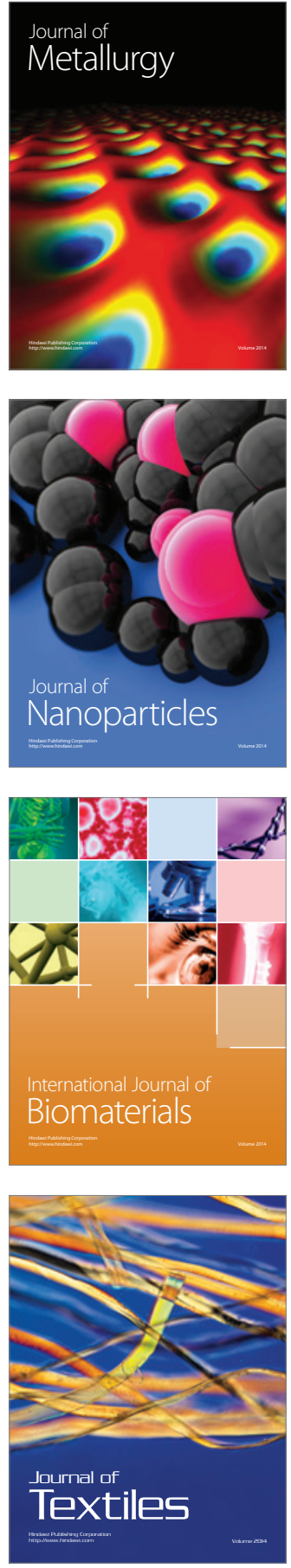